\title{
Repeated stimulation of the posterior parietal cortex in patients in minimally conscious state: A sham-controlled randomized clinical trial
}

Patients in unresponsive wakefulness syndrome (UWS - recovery of eyes opening while no behavioral sign of consciousness [1]) and in minimally conscious state (MCS - recovery of reproducible purposeful behaviors [2]) have no access to conventional rehabilitation program, apart from pharmacological or passive rehabilitation treatments. A few clinical controlled trials using transcranial direct current stimulation (tDCS) applied over the prefrontal cortex have shown promising results in patients in MCS [3-5]. tDCS has the advantage of being safe and easily performed at patient's bedsides [6]. In the present study, we decided to stimulate the posterior parietal cortex in subacute and chronic patients in MCS since, based on the literature, it is a critical hub for consciousness recovery (for a review see Ref. [7]). We hypothesized that, by increasing the excitability of this critical region, patients might recover some signs of consciousness.

Thirty-seven patients in MCS, who were 18 years or older, more than a month post injury, in stable condition and free of sedative drug(s), were in enrolled in the study. Exclusion criteria were: non-acquired brain injury, presence of metallic cerebral implant, pacemaker or any contradiction to tDCS and premorbid neurological condition. During the trial, medication and rehabilitation were kept unchanged. No patients were under any CNS-active medication. The study was approved by the ethics committee and written informed consents were obtained from patients' legal representative (ClinicalTrials.gov NCT02702362). Each patient received both active and sham tDCS sessions in a randomized order separated by 5 days of washout. tDCS was applied with a current intensity of $2 \mathrm{~mA}$ for 20 minutes per day for 5 consecutive days (at the same period of the day) with the anode positioned over the posterior parietal cortex (i.e., Pz) and the cathode placed over the right supraorbital region (DC Stimulator Plus, NeuroConn GmbH, Germany). For the sham session, the current was applied for 5 seconds at the beginning and at the end of stimulation. tDCS effects were assessed by means of standardized Coma Recovery Scale-Revised (CRS-R) assessments [8], directly before the first session (baseline) and after each active and sham tDCS session as well as five days later to evaluate the potential long-term effects. The assessor was blinded from the given treatment. As it is a crossover study, we tested the period and interaction effect using the methods described by Altman using a Mann-Whitney $U$ test [9]. The treatment effect was analyzed based on the modification of the CRS-R total scores using a Wilcoxon signed-rank test. The differences considered in the present study were: [day 5 - baseline] and [day 10 - baseline]. The effect size was calculated using the following expression $r=z / \sqrt{ } 2 n$ where $z$ is the statistics obtained from the Wilcoxon signed-rank test. Results were considered significant at $\mathrm{p}<0.05$ (for details regarding the analysis see Ref. [3]). We also identified tDCS-responders (i.e., showing new sign(s) of consciousness that was never observed before, lasting at least 5 days after the end of the stimulation, as described elsewhere [3]), and compared both groups (active and sham) using a Fisher's exact test.

Thirty-seven patients in MCS were enrolled int this trial and thirty-three completed the study (mean age $57 \pm 11$ years; 13 women; interval $6 \pm 5$ months; 20 post-traumatic). Four patients dropped-out because of medical complication (unrelated to tDCS) or due to a transfer to another facility. Demographic data and CRS$\mathrm{R}$ scores are reported in Supplementary Table 1 . All patients tolerated tDCS well and no severe side-effects related to tDCS were observed. No period or interaction effects were observed ( $p>$ 0.05 ) at day 5 or at day 10 . We also compared baseline of the second session with baseline of the first session to assess carry-over effects; we did not find any difference $(p=0.28)$. Therefore, we could calculate the treatment effect and found a significant improvement at day 5 ( $\mathrm{p}=0.012$ - effect size: 0.31$)$, while no treatment effect was identified 5 days after the last stimulation (day $10-\mathrm{p}=0.135$ ) (see Fig. 1). We found a significant difference between responders' rate (active and sham sessions $-p=0.04$, Fisher's exact test): a total of 9 (27\%) patients showed improvements during the active session (i.e., tDCS-responders), compared to 2 patients during the sham session. Four patients recovered reproducible and 1 systematic command following, 1 patient regained an intentional communication, 2 recovered visual pursuits, 1 regained the ability to recognize objects and 1 recovered intelligible verbalization.

The present study shows that tDCS over the posterior parietal cortex improves the recovery of clinical sign(s) of consciousness in some patients in MCS; however, the effects did not last when reassessed at 5-day follow-up. When comparing the effect sizes ( 0.43 vs 0.31 ), the number of responders ( 56 vs $27 \%$ ) and the duration of the effect with our previous study on tDCS targeting the left prefrontal cortex (using the same protocol [3]), we found stronger effects for the prefrontal stimulation. Stimulating the posterior parietal cortex might influence cortico-cortical and cortico-thalamic connectivity, which are both degenerated in patients with DOC [3]. On the other hand, prefrontal stimulation may induce a 


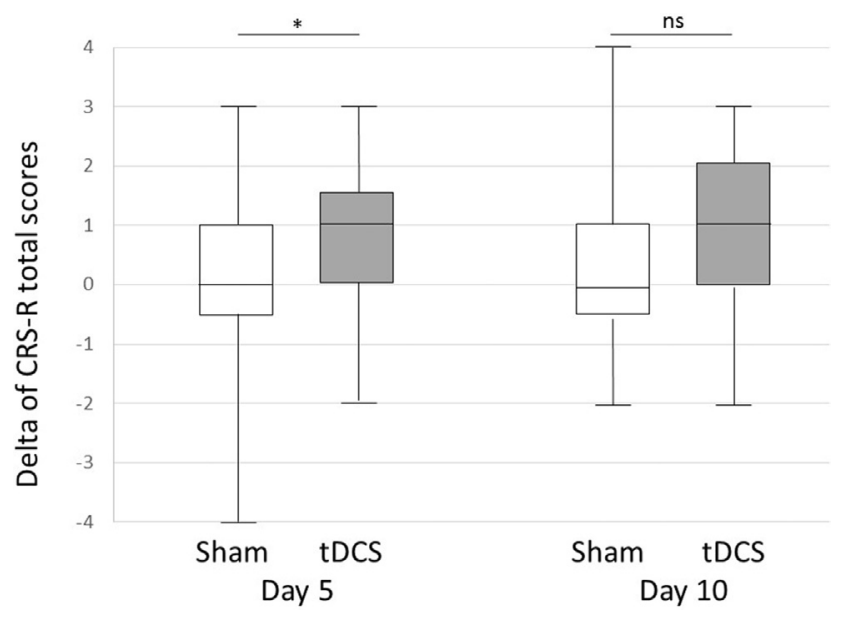

Fig. 1. Boxplot of active tDCS (in grey) and sham tDCS (in white). Treatment (day 5 $\mathrm{p}=0.012$ ) and long-term effect (day 10, i.e., 5 days after the end of the last stimulation, $\mathrm{p}=0.135$ ) were assessed using Wilcoxon match-paired signed-rank test depending on the changes of CRS-R total scores. Black lines represent the medians of the delta of the CRS-R total score between baseline and after tDCS (anodal or sham); boxes represent the interquartile range; the bars represent minimum and maximum. ${ }^{*} \mathrm{p}<0.05$.

stronger effect on the connectivity between the prefrontal cortex and the thalamus since this region has high connectivity with the striatum [10]. Through this fronto-striatal connection, a stimulation of the prefrontal cortex may reduce the inhibition of the thalamus and therefore reinforce thalamo-cortical connectivity. Though large cohort studies comparing different sites of stimulation are needed to confirm the possible superiority of one specific montage, it seems that prefrontal tDCS induces higher effect than tDCS applied over the posterior parietal cortex in patients in MCS. It is also important to note that some patients, based on their brain lesions, may benefit from tDCS targeting different cortical areas.

These results lead to several follow-up questions and future studies: 1) Was the difference between responders and nonresponders associated with anatomical differences induced by the mechanism of brain lesions? 2) Does prefrontal tDCS induce a larger magnitude of effects or some participants would respond better to prefrontal tDCS and others to parietal tDCS? 3) Can parameters of tDCS be optimized to induce larger and more long-lasting effects? 4) Can tDCS improve brain activity as measured by neuroimaging tools?

\section{Funding}

This research was supported by the National Natural Science Foundation of China (KG14086, KG08027, KG13007), the National High Technology Research and Development Program ("863"Program) of China (2015AA020514), Hangzhou Normal University (JTAS2011-01-016), the James S. McDonnell Foundation (JSMF) programs, the Belgian American Educational Foundation (BAEF), the Belgian National Fund for Scientific Research, the Wallonie-Bruxelles International (WBI), the Leon Fredericq Foundation, and the LUMINOUS project (EU-H2020-FETOPENGA686764).

\section{Conflicts of interest}

None.

\section{Acknowledgments}

We thank Emily Ohrtman for proofreading the manuscript.

\section{Appendix A. Supplementary data}

Supplementary data related to this article can be found at http:// dx.doi.org/10.1016/j.brs.2017.02.001.

\section{References}

[1] Laureys S, Celesia GG, Cohadon F, Lavrijsen J, León-Carrión J, Sannita WG, et al. Unresponsive wakefulness syndrome: a new name for the vegetative state or apallic syndrome. BMC Med 2010;8:68.

[2] Giacino JT, Ashwal S, Childs N, Cranford R, Jennett B, Katz DI, et al. The minimally conscious state definition and diagnostic criteria. Neurology 2002;58: 349-53.

[3] Thibaut A, Bruno MA, Ledoux D, Demertzi A, Laureys S. tDCS in patients with disorders of consciousness: sham-controlled randomised double blind study. Neurology 2014;82:1112-8.

[4] Thibaut A, Thibaut A1, Wannez S, Donneau AF., Gosseries O, Chatelle C, Bruno MA, Laureys S. Controlled clinical trial of repeated left prefrontal transcranial direct current stimulation in patients with chronic minimally conscious state. 19th Association for the Scientific Study of Consciousness meeting; Paris, France.

[5] Angelakis E, Liouta E, Andreadis N, Korfias S, Ktonas P, Stranjalis G, et al Transcranial direct current stimulation effects in disorders of consciousness. Arch Phys Med Rehabil 2014;95(2):283-9.

[6] Bikson M, Grossman P, Thomas C, Zannou AL, Jiang J, Adnan T, et al. Safety of transcranial direct current stimulation: evidence based update 2016. Brain Stimul 2016;9(5):641-61.

[7] Laureys S, Schiff ND. Coma and consciousness: paradigms (re)framed by neuroimaging. Neuroimage 2012;61(2):478-91.

[8] Di H, He M, Zhang Y, Cheng L, Wang F, Nie Y, et al. Chinese translation of the coma recovery scale-revised. Brain Inj 2017 Jan 26:1-3. http://dx.doi.org/ 10.1080/02699052.2016.1255780 [Epub ahead of print].

[9] Altman DG. In: Chapman, Hall/CRC, editors. No Title. London: Practical statistics for medical research; 1990. p. 467-71.

[10] Lant ND, Gonzalez-Lara LE, Owen AM, Fernández-Espejo D. Relationship between the anterior forebrain mesocircuit and the default mode network in the structural bases of disorders of consciousness. Neuroimage Clin 2016;10:27-35.

Wangshan Huang International Vegetative State and Consciousness Science Institute, Hangzhou Normal University, Hangzhou, China E-mail address: huangwangshan@sina.cn.

Sarah Wannez

Coma Science Group, GIGA Research, Cyclotron Research Centre and Neurology Department, University and University Hospital of Liège,

Belgium

E-mail address: sarah.wannez@ulg.ac.be.

Felipe Fregni Spaulding Neuromodulation Center, Spaulding Rehabilitation Hospital, Harvard Medical School, Boston, MA, USA E-mail address: felipe.fregni@gmail.com.

Xiaohua Hu, Shan Jing Department of Rehabilitation, Hangzhou Wujing Hospital, Hangzhou, China E-mail addresses: huxiaohua-hz@qq.com (X. Hu), dr_jingshan@163.com (S. Jing).

Geraldine Martens Coma Science Group, GIGA Research, Cyclotron Research Centre and Neurology Department, University and University Hospital of Liège, Belgium E-mail address: geraldine.martens93@gmail.com. 
Minhui He, Haibo Di* International Vegetative State and Consciousness Science Institute, Hangzhou Normal University, Hangzhou, China

Steven Laureys

Coma Science Group, GIGA Research, Cyclotron Research Centre and Neurology Department, University and University Hospital of Liège, Belgium

E-mail address: steven.laureys@ulg.ac.be.

Aurore Thibaut ${ }^{* *}, 1$ Coma Science Group, GIGA Research, Cyclotron Research Centre and Neurology Department, University and University Hospital of Liège, Belgium

Spaulding Neuromodulation Center, Spaulding Rehabilitation Hospital, Harvard Medical School, Boston, MA, USA
* Corresponding author. International Vegetative State and Consciousness Science Institute, Hangzhou Normal University, 18 Xuelin Road, Xiasha Higher Education Campus, Hangzhou, China.

** Corresponding author. Spaulding Neuromodulation Center, Spaulding Neuromodulation Center, 79/96 13th Street, 02129 Charlestown, MA, United States. E-mail addresses: heminhui2003@aliyun.com (M. He), dihaibo19@aliyun.com (H. Di). E-mail address: athibaut@neuromodulationlab.org (A. Thibaut).

2 February 2017 Available online $\mathrm{xxx}$

\footnotetext{
1 shared last authors.
}

Please cite this article in press as: Huang $\mathrm{W}$, et al.Repeated stimulation of the posterior parietal cortex in patients in minimally conscious state: A sham-controlled randomized clinical trial, Brain Stimulation (2017), http://dx.doi.org/10.1016/j.brs.2017.02.001 